\title{
Smart Grid Interoperability Use Cases for Extending Electricity Storage Modeling within the IEC Common Information Model
}

\author{
Nigel Hargreaves \\ Brunel University, National Grid \\ nigel.hargreaves@brunel.ac.uk
}

\author{
Gareth Taylor \\ Brunel University \\ gareth.taylor@brunel.ac.uk
}

\author{
Alex Carter \\ National Grid \\ alex.carter@nationalgrid.com
}

\begin{abstract}
The IEC Common Information Model (CIM) is recognized as a core standard, supporting electricity transmission system interoperability. Packages of UML classes make up its domain ontology to enable a standardised abstraction of network topology and proprietary power system models. Since the early days of its design, the CIM has grown to reflect the widening scope and detail of utility information use cases as the desire to interoperate between a greater number of systems has increased. The cyber-physical nature of the smart grid places even greater demand upon the CIM to model future scenarios for power system operation and management that are starting to arise. Recent developments of modern electricity networks have begun to implement electricity storage (ES) technologies to provide ancillary balancing services, useful to grid integration of large-scale renewable energy systems. In response to this we investigate modeling of grid-scale electricity storage, by drawing on information use cases for future smart grid operational scenarios at National Grid, the GB Transmission System Operator. We find current structures within the CIM do not accommodate the informational requirements associated with novel ES systems and propose extensions to address this requirement.
\end{abstract}

Index Terms-Common Information Model, CIM, interoperability, smart grid, energy storage.

\section{INTRODUCTION}

\section{A. The Common Information Model}

The IEC Common Information Model (CIM), IEC $61970 / 61968$, [1] is regarded as central to a number of national and international smart grid standards frameworks [2] and forms the basis of one of five core smart grid interoperability standards identified by the National Institute of Standards and Technology (NIST) [3]. The CIM provides an object-oriented, scalable and extensible semantic model for modeling power system resources. IEC61970 was originally intended as a common information model to map energy management system application programming interfaces (EMS-API) for transmission system operation but has grown to become a power utility domain ontology [4]. Successive releases of the CIM, driven by power utility information use cases, are continuously increasing its scope and resolution to support smart grid scenarios for power system interoperability and management [5]. Examples of such developments include modeling of time-variable phenomena through the use of incremental data models and forthcoming extensions for Diagram Layout and Environmental Data [6].

\section{B. Future Operational Scenarios}

In contrast to the deterministic nature of traditional energy despatch, future operational scenarios proposed by National Grid anticipate supply and demand to be increasingly probabilistic leading to stochastic energy forecasting by Transmission System Operators (TSOs). Such complexity can be attributed to a range of factors; these include, large weather-dependent renewable energy injections, unmetered (by the TSO) embedded generation and greater demand variability from consumers responding to time of use tariffs through advanced metering infrastructures (AMIs), as well as large-scale use of electric vehicles and heat pumps. TSO control over system frequency will therefore become challenged by the increased variability of generation, demand and interconnector flows, coupled with the reduction in system inertia as a consequence of greater proportions of asynchronous wind generation and DC interconnection. Some of the factors affecting future energy scheduling are visualized in Fig. 1.

National Grid have identified the need for doubling the amount of Operating Reserve (including STOR) on the GB network from $4 \mathrm{GW}$ to $8 \mathrm{GW}$ to address frequency control due to greater variability of network in-flows [7]. There is also an emerging pattern to limit voltage gain more often, especially at times of large injections of wind energy during periods of minimum demand, such as at night in summer, in the UK.

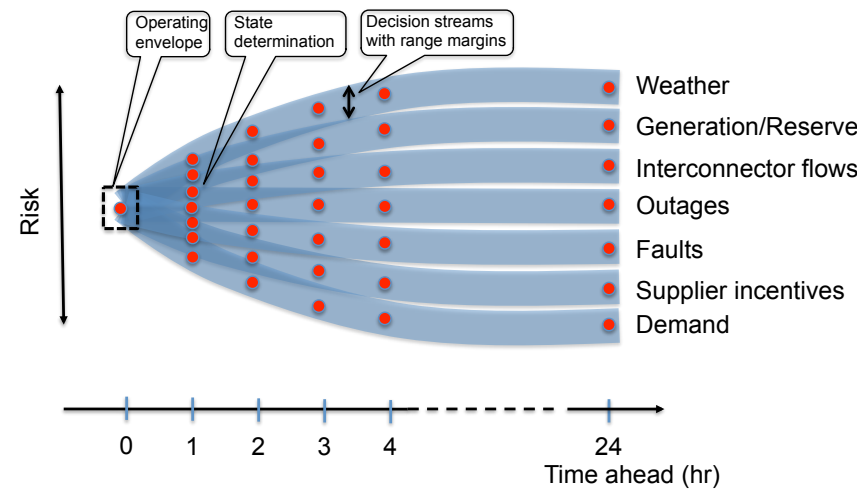

Fig. 1. Illustration of stochastic nature of energy scheduling within multi-factorial scenario forecasting to derive a network operational envelope for real time. 


\section{The Case for Extending the CIM for Energy Storage}

Energy storage is not unfamiliar in electrical networks but the perspectives taken in this paper need to be qualified for comparison with the IEC CIM. For example, the stored energy in biomass, reservoirs, coal piles, gas and oil depots, is within the perspective of energy security where sustainability of generation is the focus at times of disruption to energy supply. Whereas ES technologies, such as pumped storage, batteries and flywheels are within the perspective of decarbonisation, where generation flexibility and demandsupply balancing are the focus, under high levels of renewable energy penetration. Both perspectives affect realistic operational scenarios that the controllers of smart grids and their information resources must address [8][9].

These perspectives offer an incomplete picture of ES technologies as there are further examples that may be considered, including specialist applications, such as superconducting magnetic energy storage (SMES) [10] and the molten metal heat stores used within solar-thermal power stations [11]. In addition, general applications such as aggregated amounts of domestic heat storage, heat pumps and battery-electric vehicle storage will be of interest to TSOs within the context of the future smart grid [12]. This perspective reflects one way the IEC CIM could evolve to capture the changing information needs of smart grid network operators. The need to manage more variable supply and demand, distributed over a wider geographic area has called for smarter, more flexible electricity networks. Thus the role of the distribution network can no longer simply be regarded from the transmission side as load. New forms of aggregated flexible load embedded within distribution networks, as mentioned above, will be significant to TSOs. Additionally, the transmission network can no longer be only regarded as supply through its connections to large generators from the perspective of a Distribution Network Operator (DNO) due to the proliferation of embedded generation. As new forms of energy storage are implemented in increasing size and number within both of these domains as well, they present the need to be modeled within the CIM, in addition to the established pumped storage technologies.

\section{USE CASES FOR ENERGY STORAGE SYSTEMS}

An understanding of the use cases for different forms of energy storage systems is necessary to drive the information about them that should be modeled within the IEC CIM. We will look at examples of ES deployment in this section and gather the characteristics that will form attributes of the proposed information model extensions. In Section III we will address the current and proposed UML class structures for ES information modeling before proceeding to our Conclusions and Future Work in sections IV and V.

Storage may be regarded as both a generator and a load where different technologies offer scalable and over-lapping performance influenced by cost and physical constraints. From the perspective of decarbonisation, use cases for pumped hydro, batteries and flywheel energy storage systems in the USA and Japan and their operational characteristics are presented in [13]-[17]. Examples of other advanced installations include the pilot $200 \mathrm{kWh} / 600 \mathrm{~kW}$ battery deployment by UK Power Networks for wind farm voltage stability [18]; a 4MWh/46MVA Ni-Cd battery outage support installation in Alaska [19]; a $2.5 \mathrm{MWh} / 300 \mathrm{~kW}$ cryogenic air storage and cogeneration plant using waste heat in the UK [20]; a proposed $4 \mathrm{GWh} / 1 \mathrm{GW} \mathrm{NaS}$ battery voltage and outage support facility in Texas [21]. In general, as the penetration of renewable energy sources increases the value and utility of appropriately sized ES also increases due to its flexibility and responsiveness to network dynamics [22]. Significantly, the use of $\mathrm{NaS}$ battery technology to stabilize and back-up up large windfarms is developing as a way of aligning deliverable energy with forecasts [23], [24]. Such developments could displace the need for some thermal spinning reserve, saving the cost and emissions that would otherwise be due to operating large generators in this inefficient manner.

Fast response storage technologies like flywheels, batteries and some pumped storage, able to discharge power and recharge rapidly to follow regulation signals are deployed as ancillary services for voltage and frequency correction for power quality and stability. Massively scaled battery installations are also being deployed for demand support in the absence of a suitable geography for pumped technologies. Arbitrage is a further use case for ES with sufficient energy capacity, presenting an attractive way to avoid costs associated with renewable energy curtailment. It may also help to prevent the high investment required to reinforce transmission lines if it were implemented at load centres and used to load-shift from peak to off-peak times.

Energy storage technologies have overlapping operational envelopes. A simplified representation of how this may look for the most prevalent ES technologies is presented in Fig. 2. However it is important to recognize that the shape drawn below for a given ES technology changes with scaling. For information modeling purposes therefore, our

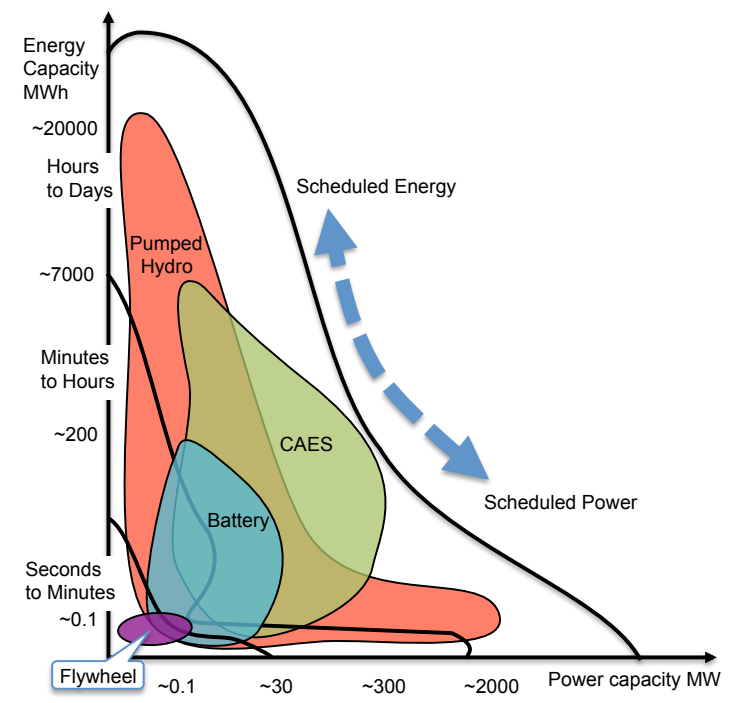

Fig. 2. A representation of how different energy storage technologies may deploy relative to their different energy and power capacities and their discharge operational window (not to scale). Based on data found in [14]. 
characterization of ES technologies is based on energy capacity, power capacity, responsiveness to discharge/charging and discharge/charge duty duration, corresponding to the following use cases:

- Scheduled energy (despatchable generation, outage support)

- $\quad$ Regulating reserve (voltage and frequency control, power quality)

- $\quad$ Arbitrage (load-shifting, demand support)

This approach prepares us for the design of the Energy Storage Model (ESM) in UML, by pointing to the requisite class objects and their attributes.

\section{CURRENT AND PROPOSED UML CLASS STRUCTURES}

In a current version of IEC 61970 (CIM 15) [1] energy storage is represented by the models for Pumped Hydro and Compressed Air Energy Storage (CAES). These technologies, found within the Production Package, are shown in the simplified UML class diagram below (Fig. 3) in relation to the "GeneratingUnit" class. "GeneratingUnit" generalizes a grouping of different kinds of synchronous machines that act as generators, or as a "HydroPump" for charging pumped hydro reservoirs. The "SynchronousMachine" association to "GeneratingUnit" also generalizes to a "RotatingMachine" class (found in the Wires Package) to provide for the use case of regulation of voltage and frequency. Observing the difference between the use of synchronous machines and the way in which other ES technologies (such as batteries and flywheels) convert energy, we propose a new Package as a template to extend the IEC CIM to accommodate other forms of energy storage in preparation for smart grid information requirements.

Our approach reuses existing CIM UML classes and stereotypes as far as possible in order to minimize the amount of code extension to build a data model in CIM RDF XML. Concrete classes representing different battery types and flywheels are contained within abstract containers linked to operational data curves, generally following the stereotypes already provided by the existing models within the IEC CIM. The proposed UML class diagram for energy storage presents

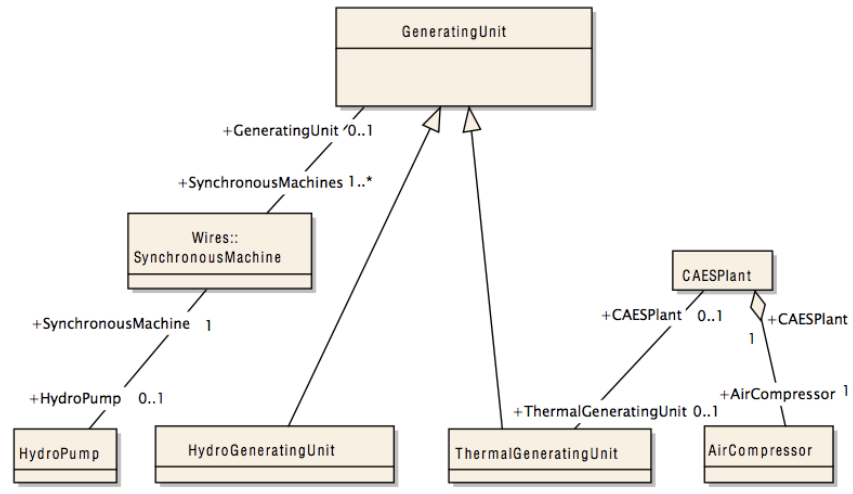

Fig. 3. Simplified existing energy storage model taken from IEC CIM 15, Production Model, "Main" diagram, [1] little detail of attributes and enumerations that would be required within the classes as our experience with these technologies within electrical grids is limited so far. We designed the ESM in Enterprise Architect [25] and have enumerated data types for the principal battery technologies (Sodium Sulphur, Lithium-ion and Vanadium redox flow), as well as including sample attributes for Flywheel "SpinSpeed" (using the existing "RotationSpeed" enumeration stereotype) and creating a new attribute, "StockPile" to indicate the volume of a fossil fuel referred to by the "FuelStore" class. However, our intention at this point is to focus mainly on the model architecture.

An explanation of the ESM (Fig. 4) logic and comparison to classes in the established Production Model now follows:

\section{A. Decarbonisation perspective}

Physical storage technologies are aggregated into the "EnergyStorageUnit" container. Attributes from the individual technology in combination with curves accessed from the "ESLevelSchedule" help to determine the power and energy level status within a particular "EnergyStorageUnit" technology. These would need to account for the number of duty cycles, the degree of discharge, energy leakage, electrolyte temperature (in the case of batteries) and performance degradation etc. for a given technology. This is similar to treatment given to calculating the potential energy of a hydro reservoir in the Hydro Model.

The role of the "EnergyStoragePlant" class is similar to the role of "HydroPowerPlant" in the way it offers a power system resource that can be used in both generation and load modes of operation. From a network-wide perspective therefore, "EnergyStoragePlant" acts as a container for distributed energy storage units connected to different parts of the grid.

EnergyStoragePlant also aggregates equipment containers for charging and discharging stored energy through the classes "ESChargingUnit" and "ESGeneratingUnit", which are loosely equivalent to the status of the "GeneratingUnit" class in the Production Model. This would be useful to the metering that would be required for storage technologies under different modes of operation, by clearly differentiating charging from viewing it as negative generation. Details of equipment that manage the charging and discharging of energy stores were not modeled but it is assumed they would aggregate to the "ESChargingUnit" and "ESGeneratingUnit" classes. Operating cost curves and the discharging and charging schedules, provide information about the optimum time and mode of operation for energy storage plant. The configuration of the charging and discharging schedules under the superclass "RegularIntervalSchedule" reflects the scheduled energy and arbitrage use cases stated above.

The remaining use case, concerning regulating reserve for frequency and voltage regulation and ancillary services for power quality, is addressed as follows: Associations from the "ESChargingUnit" and "ESGeneratingUnit" classes to an "ESReguatingUnit" class, have a multiplicity that allows ES systems to switch between charging and discharging if 


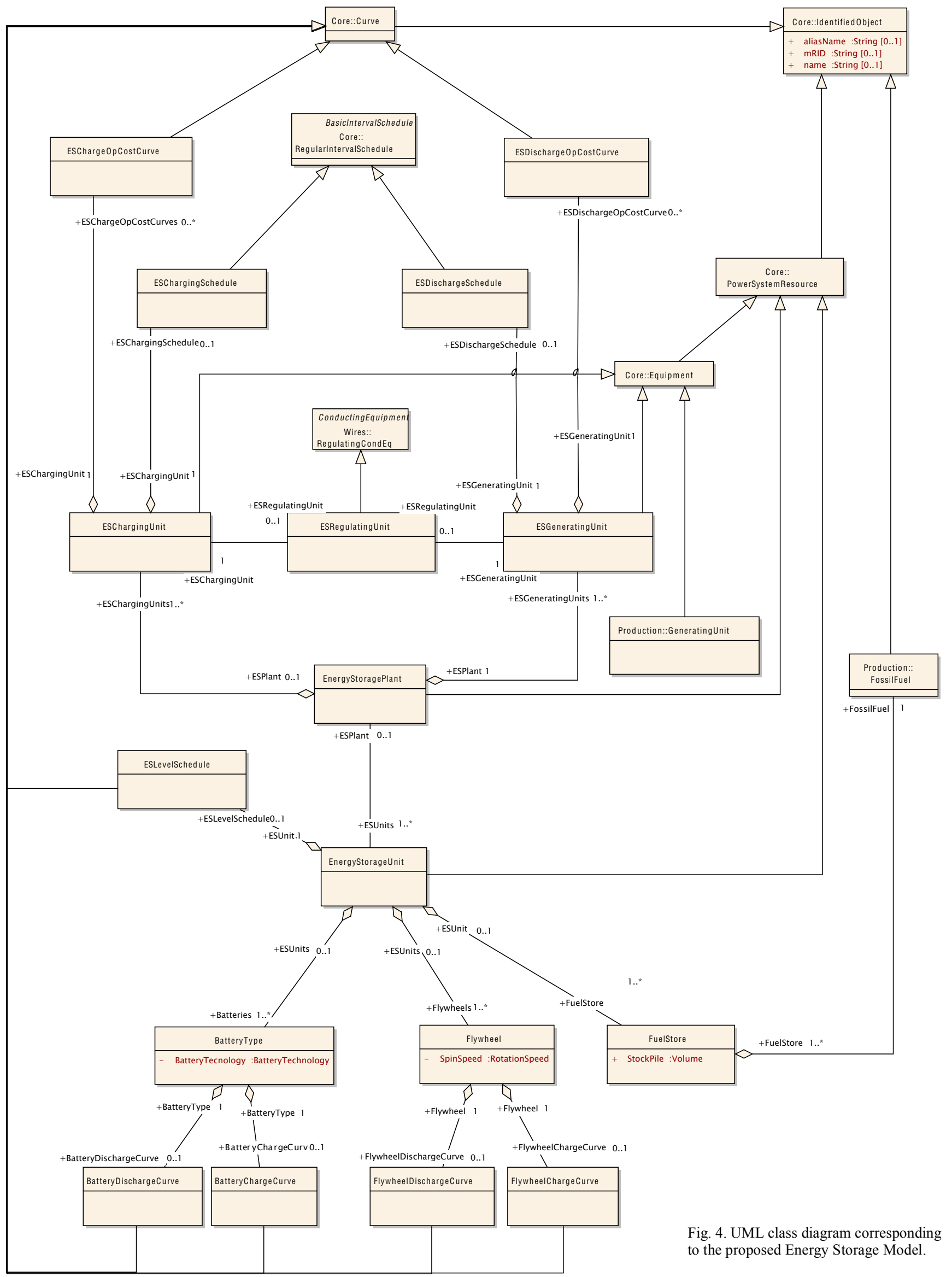


required by a regulation signal. This signal is derived in a similar way to other regulating equipment within the Wires Model through generalization of the "ESRegulatingUnit" class to the "RegulatingCondEq" superclass. The creation of the "ESRegulatingUnit" class was necessary (and follows the analogy of the "RotatingMachine" class within the Wires Package) as the associated charging and discharging classes already generalize to the "PowerSystemResource" class.

The IEC CIM modelling convention conforms to only singular generalizations from one class to another. Generalizing of the "ESRegulatingUnit" in this way, effectively connects the charging and discharging capabilities of energy storage plant alongside rotating machines, static VAr compensators, shunt compensators and frequency converting equipment, adding another tool for regulation purposes.

So far, the model has been developed to reflect the use cases for battery and flywheel energy storage technologies. But it offers an extensible information architecture to accommodate other forms of ES technology such as SMES and supercapicitors when warranted. Classes representing these technologies could be aggregated to the "EnergyStorageUnit" class with their own sets of curves and classes for any ancillary equipment. Attributes particular to a given storage technology in addition to the ones presented would also be required to meet the information requirements of power system control applications.

\section{B. Energy Security perspective}

We have described the use cases for the proposed ESM that relate to the perspective of decarbonisation. The "Reservoir" class within the Hydro Model is implicitly an example of a fuel store already modeled within the IEC CIM but we believe this approach needs extension to address the energy security use case, especially as primary fuel supply lines can no longer be assured with increasing dependence on imports. We must therefore consider the scope applied in our definition of energy security in order to justify the level of detail in our information model for a given use case. For example, we could extend the scope of energy security to include all known accessible reserves of a particular fuel type, or to extracted reserves held in the stockpiles in a geographic region, or to the stockpiles of fuels on-site at the points of conversion to electrical energy.

The inclusion of the class "FuelStore" in Fig. 4, is intended to reflect the availability of stored energy in stockpiles of fuels used by thermal generators, which reflects the latter scope for energy security. However, the "FuelStore" class could become a container to classes reflecting the greater spatial scopes for energy security mentioned above. In doing so, the natural resources and environmental services of a particular geographic region would be addressed. This may be considered to be beyond the intended scope of the IEC CIM, but is likely to be part of a national energy security strategy.

The class object "FossilFuel", already modeled in CIM15, aggregates to "FuelStore" carrying values of specific heat content for coal, gas, oil and lignite. Thus the energy content of the fuel store can be calculated from the combination of the attributes for the amount of fuel ("StockPile :Volume") and its type ("fossilFuelType"), enumerated within the "FuelStore" class. "FuelStore" also aggregates to "EnergyStorageUnit", which generalizes to "PowerSystemResource", presenting a logical information pathway to report the available energy within fuel stores accessible to thermal generating units connected to the grid.

As the ESM is designed to address issues associated with energy security, we would recommend that the Production Model Datatypes enumeration for "FuelType" be extended to include biomass, as it is already being consumed in thermal power stations. This would enable better definition of fuel consumption within co-fired power stations. Biomass is also likely to form an increasingly significant part of sustainable energy strategies in future.

\section{CONCLUSIONS}

We have highlighted the need to address interoperability use cases for energy storage systems within the IEC CIM now extends beyond its current scope from the perspectives of decarbonisation and energy security of electricity networks. The proposed ESM could serve as an extension for integrating ES systems newly appearing on electricity smart grids into the IEC CIM. It could also offer an approach to quantifying the potential energy available in reserves from an energy security standpoint. The proposed model has been designed as a separate Package that could realize a client relationship with the extant Production or Wires Packages within the CIM.

\section{FUTURE WORK}

The ESM requires the addition of further enumerations and Datatypes as attributes to describe the information requirements of the modeled energy storage systems. The authors also recognize there is a need to investigate the information modeling of distributed energy storage systems that will be embedded within future distribution networks, to include the relationship of the IEC 61850 substation automation information standard to IEC 61970 for energy storage modelling. The counterpart relationship between the use cases for grid-scale energy storage and demand reduction management also warrants further investigation from an information modeling point of view.

\section{ACKNOWLEDGEMENTS}

National Grid Electricity Transmission (NGET) working in collaboration with Brunel University have commissioned this research as part of the Brunel Industrial Doctorate Scheme (BIPS). The authors would like to acknowledge the valuable insights and suggestions for this paper offered by members of the National Grid Operational Strategy Development team: Dr. Bernie Dolan, Dr. Ronan Jamieson and Mr. Stefan Pantea and Dr. Alan McMorran of Open Grid Systems. 
The authors would like to thank National Grid Plc and the Engineering and Physical Sciences Research Council (EPSRC) for funding this research project. This paper was published with permission from National Grid Plc. The opinions expressed in this paper are that of the authors and not that of National Grid.

\section{REFERENCES}

[1] CIM Users Group, "Draft 61970cim15v32_61968cim11v13". http://cimug.ucaiug.org/CIM\%20Model\%20Releases/Forms/AllItems.a spx, 2011.

[2] M. Uslar, M. Specht, S. Rohjans, J. Trefke, J. M. Vasquez Gonzalez, "Examples of Using the CIM," Springer-Verlag, Berlin Heidelberg, 2012.

[3] National Institute of Standards and Technology (NIST), "NIST Identifies Five 'Foundational' Smart Grid Standards". http://www.nist.gov/public_affairs/releases/smartgrid_100710.cfm 2010.

[4] N. Hargreaves, G. Taylor, A. Carter, "Information Standards to support Application and Enterprise Interoperability for the Smart Grid," in Power \& Energy Society General Meeting, 2012. PES '12. IEEE, 2012, accepted.

[5] N. Hargreaves, G. Taylor, A. Carter and A. McMorran, "Developing emerging standards for power system data exchange to enable interoperable and scalable operational modelling and analysis," Universities' Power Engineering Conference (UPEC), Proceedings of 2011 46th International, pp. 1-5, 2011.

[6] Electric Power and Research Institute (EPRI), "Research Overview", http://my.epri.com

[7] National Grid, "Gone Green: A scenario for 2020". National Grid plc, London, 2008

[8] Zhenhua Jiang, Fangxing Li, Wei Qiao, Hongbin Sun, Hui Wan, Jianhui Wang, Yan Xia, Zhao Xu and Pei Zhang, "A vision of smart transmission grids," in Power \& Energy Society General Meeting, 2009. PES '09. IEEE, 2009, pp. 1-10.

[9] G. Strbac, M. Black, "Future value of storage in the UK. Final report". Centre for Distributed Generation and Sustainable Electrical Energy, University of Manchester, Imperial College, University of Strathclyde, 2004.

[10] M. H. Ali, Bin Wu and R. A. Dougal, "An Overview of SMES Applications in Power and Energy Systems," Sustainable Energy, IEEE Transactions on, vol. 1, pp. 38-47, 2010.

[11] D. N. Harries, M. Paskevicius, D. A. Sheppard, T. E. C. Price and C. E. Buckley, "Concentrating Solar Thermal Heat Storage Using Metal Hydrides," Proceedings of the IEEE, vol. 100, pp. 539-549, 2012.

[12] Y. Ma, T. Houghton, A. Cruden and D. Infield, "Modeling the Benefits of Vehicle-to-Grid Technology to a Power System," Power Systems, IEEE Transactions on, vol. PP, pp. 1-1, 2012.
[13] J. P. Barton and D. G. Infield, "Energy storage and its use with intermittent renewable energy," Energy Conversion, IEEE Transactions on, vol. 19, pp. 441-448, 2004.

[14] C. E. T. Foote, A. J. Roscoe, R. A. F. Currie, G. W. Ault and J. R. Mcdonald, "Ubiquitous energy storage," in Future Power Systems, 2005 International Conference on, 2005, pp. 6 pp.-6.

[15] Bo Yang, Y. Makarov, J. Desteese, V. Viswanathan, P. Nyeng, B. McManus and J. Pease, "On the use of energy storage technologies for regulation services in electric power systems with significant penetration of wind energy," in Electricity Market, 2008. EEM 2008. 5th International Conference on European, 2008, pp. 1-6.

[16] S. Yeleti and Yong Fu, "Impacts of energy storage on the future power system," in North American Power Symposium (NAPS), 2010, 2010, pp. 1-7.

[17] W. F. Pickard and D. Abbott, "Addressing the Intermittency Challenge: Massive Energy Storage in a Sustainable Future [Scanning the Issue]," Proceedings of the IEEE, vol. 100, pp. 317-321, 2012.

[18] ABB, "Energy storage - smoothing the transition to smarter grids". 2011.

http://www.abb.com/cawp/seitp202/4ec961176b95a792c125793100300 be8.aspx

[19] ABB Case note, "World's Largest Battery Energy Storage System. Fairbanks, Alaska." (PDF).

http://www05.abb.com/global/scot/scot232.nsf/veritydisplay/3c4e1581 6e4a7bf1c12578d100500565/\$file/case_note_bess_gvea_fairbanksweb.pdf

[20] J. St.John, "Liquid Air for Utility-Scale Energy Storage?" Greentechmedia, 2012. http://www.greentechmedia.com/articles/read/liquid-air-for-utilityscale-energy-storage/

[21] J. Kumagai, "A Battery as Big as the Grid". IEEE Spectrum Inside Inside Technology, 2012. http://spectrum.ieee.org/energy/the-smarter-grid/a-battery-as-big-asthe-grid

[22] Y. V. Makarov, Pengwei Du, M. C. W. Kintner-Meyer, Chunlian Jin and H. F. Illian, "Sizing Energy Storage to Accommodate High Penetration of Variable Energy Resources," Sustainable Energy, IEEE Transactions on, vol. 3, pp. 34-40, 2012.

[23] N. Kawakami, Y. Iijima, Y. Sakanaka, M. Fukuhara, K. Ogawa, M. Bando and T. Matsuda, "Development and field experiences of stabilization system using $34 \mathrm{MW}$ NAS batteries for a $51 \mathrm{MW}$ wind farm," in Industrial Electronics (ISIE), 2010 IEEE International Symposium on, 2010, pp. 2371-2376.

[24] A. D. Shakib and G. Balzer, "Energy storage design and optimization for power system with wind feeding," in Probabilistic Methods Applied to Power Systems (PMAPS), 2010 IEEE 11th International Conference on, 2010, pp. 54-59.

[25] Sparx Systems, "Enterprise Architect v9.3 Corporate Edition". http://www.sparxsystems.com 